\title{
Recurrent Metastatic High-Grade Osteosarcoma: Disease Stabilization and Successful Pregnancy Outcome following Aggressive Multimodality Treatment
}

\begin{abstract}
Prognosis for refractory/recurrent metastatic osteosarcoma (OS) remains dismal with 3-year survival rates $<20 \%$. Achievement of more than 5 years of stable, refractory/recurrent metastatic OS disease in our patient is itself unique and attributable to multimodality therapy. Her high-dose chemotherapy regimen with alkylating agents did not lead to infertility, making her case even more unique. Successful disease stabilization and pregnancy outcome in our patient with metastatic multiple relapsed OS is one of the first cases published. We need further understanding and exploration of pathogenesis, chemoresistant mechanisms, and multimodality therapies including immunotherapy for OS.
\end{abstract}

Keywords: Cancer therapy, fertility, osteosarcoma

\section{Introduction}

Osteosarcoma (OS) is the most common primary malignant bone tumor in children and adolescents, characterized by malignant disorganized osteoblastic differentiation and commonly occurring in the metaphysis of long bones. Metastatic disease is associated with 3-year overall survival of $<20 \%$ compared to $60 \%-70 \%$ in localized disease. Recurrent OS occurs in almost half of patients presenting with localized disease and in $80 \%$ of patients presenting with metastatic disease. Complete surgical resection, the most important determinant of outcome in relapsed patients, only yields 3- to 5-year survival rates of $20 \%$ $40 \%$. Incomplete resection following recurrence leads to very poor prognosis and is invariably fatal. ${ }^{[1-5]}$ Here, we report a patient with metastatic multiple relapsed OS who had successful pregnancy outcome and long-term event-free survival after multimodality treatment.

\section{Case Report}

High-grade OS of the right distal femur was diagnosed in a 16-year-old Latino girl. Pathology revealed hyperchromatic and osteoblastic tumor cells with pleomorphism and osteoid deposition. Chest computed

This is an open access journal, and articles are distributed under the terms of the Creative Commons Attribution-NonCommercial-ShareAlike 4.0 License, which allows others to remix, tweak, and build upon the work non-commercially, as long as appropriate credit is given and the new creations are licensed under the identical terms.

For reprints contact: reprints@medknow.com tomography (CT) identified three small $(<3 \mathrm{~mm})$ pulmonary nodules at initial presentation. Diagnosis of high-grade OS with resectable pulmonary metastases made her eligible for treatment per Children's Oncology Group protocol AOST0331 with high-dose methotrexate, adriamycin, and cisplatin (MAP).

After two cycles of MAP, the extremity tumor was completely resected with wide margins and metallic prosthesis was implanted. Pathology revealed poor response to prior chemotherapy with $65 \%$ necrosis of the tumor cells. In addition, her restaging $\mathrm{CT}$ scan revealed the progression of her pulmonary disease. She was taken off protocol therapy and given seven cycles of high-dose ifosfamide and etoposide followed by excision/wedge resection of eight new pulmonary lesions. Complete remission was confirmed at the end of therapy.

Relapse occurred within a year with the appearance of three left-sided pulmonary nodules confirmed to be metastatic OS by biopsy. Surgeons were unable to attain complete resection of these lesions due to the proximity of the nodules to the aorta and heart. Two cycles of adjuvant chemotherapy with gemcitabine and taxotere were given. Persistent nodular growth led to the left

\footnotetext{
How to cite this article: Khurana M, Gruner B, Sathi BK. Recurrent metastatic high-grade osteosarcoma: Disease stabilization and successful pregnancy outcome following aggressive multimodality treatment. Indian J Med Paediatr Oncol 2018;39:530-2.
}

\section{Monica Khurana ${ }^{1,2}$, Barbara Gruner ${ }^{3}$, Bindu Kanathezhath Sathi ${ }^{1,3}$}

${ }^{1}$ Department of Hematology/Oncology, UCSF Benioff Children's Hospital Oakland, Oakland, California 94609, ${ }^{2}$ Department of Hematology/Oncology, Indiana University School of Medicine, Riley Hospital for Children at Indiana University Health, Indianapolis, Indiana 46202, ${ }^{3}$ Department of Pediatric Hematology/Oncology, University of Missouri School of Medicine, Columbia, Missouri 65201, USA

Address for correspondence: Dr. Bindu Kanathezhath Sathi, Department of Pediatric Hematology/Oncology, University of Missouri School of Medicine, 404 N Keene Street, Columbia, Missouri 65201, USA.

E-mail:sathib@health.missouri. edu

Access this article online

Website: www.ijmpo.org

DOI: 10.4103/ijmpo.ijmpo_83_17 Quick Response Code:

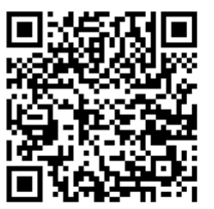


main pulmonary artery obstruction and local destruction of the $6^{\text {th }}$ and $7^{\text {th }}$ ribs. Palliative rib radiation $(3000 \mathrm{cGy}$ at 300 cGy in 10 fractions) ameliorated local pain, shortness of breath, and cough [Figure 1].

With only minimal reduction in size of the pulmonary nodules [Figure 2a], she and her family opted for Phase I investigational therapy at the National Cancer Institute and enrolled on the Pediatric Oncology Branch protocol 07-C-0040. Therapy consisted of intravenous infusions every 2 weeks with lexatumumab. After 22 cycles of lexatumumab (2 infusions per cycle), disease stabilized with calcified, necrotic tumor [Figure 2b] and positron emission tomography (PET) scan was negative for fluorodeoxyglucose-avid tumor; thus, she was deemed to have stable disease.

At 22 years of age, she became pregnant. Due to gestational diabetes mellitus and persistent calcified thoracic nodules, she was referred to the high-risk obstetrics team. She gave birth to a healthy baby boy through elective cesarean section without any perinatal complication.

Surveillance for disease progression continued every 6 months until she was lost to follow-up 2 years ago. At the time of her last clinic visit, the patient remained clinically asymptomatic and had stable disease per PET CT imaging, more than 10 years after initial presentation and more than 3 years after lexatumumab.

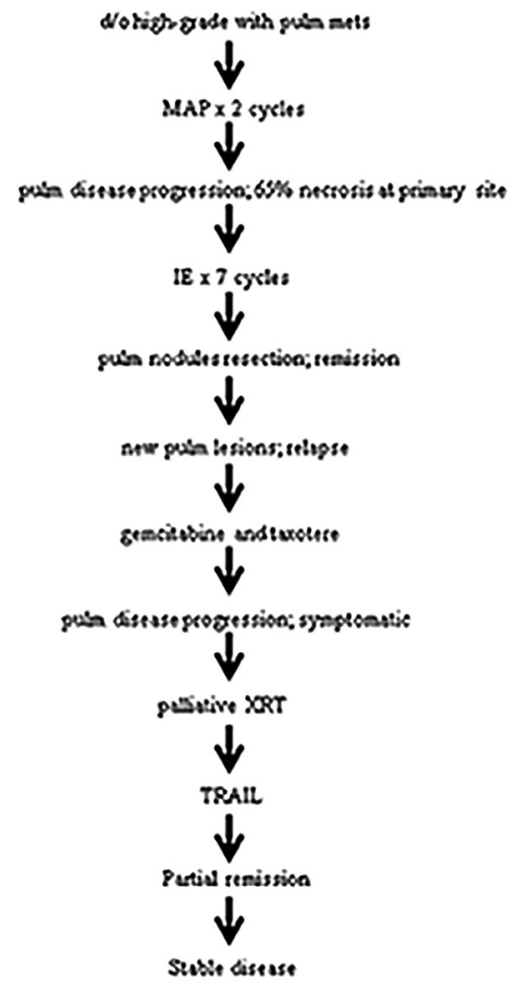

Figure 1: Treatment course. Abbreviations: d/o: Diagnosis of; PP: Pulm pulmonary; MAP: Methotrexate, adriamycin, and cisplatin; IE: Ifosfamide etoposide; XRT: Radiation; TRAIL: Tumor necrosis factor-related apoptosis-inducing ligand

\section{Discussion}

The prognosis of recurrent metastatic high-grade OS is extremely poor, with $<10 \%$ overall survival. Overall survival is even lower when complete surgical resection is not possible. Poor response to neoadjuvant chemotherapy, early relapse (relapse $<2$ years after initial complete resection), and incomplete surgical resection of pulmonary metastasis placed this patient at very high risk for further disease progression. Our patient has been doing well 5 years after completion of all therapy and presents a unique scenario where the addition of newer biological agents to conventional chemoradiation therapy potentially led to disease stabilization. Prolonged disease stabilization, in this case, is attributable to lexatumumab, which mimics the natural tumor necrosis factor-related apoptosis-inducing ligand molecule that activates cell death receptors to induce apoptosis. The effects of tumor cell apoptosis were augmented by radiation therapy before the administration of lexatumumab, as previously noted by Merchant et al. ${ }^{[6]}$ Several Phase I and II trials using newer biological agents are underway for recurrent or refractory OS, some more successful than others in prolonging remission. This includes monoclonal antibodies against insulin-like growth factor-1 receptor (cixutumumab), histone deacetylase inhibitors, mTOR inhibitors such as everolimus, telomerase inhibitors such as imetelstat, and antiangiogenic agents such as bevacizumab and sorafenib. ${ }^{[7-13]}$

Such life-saving treatments are not without risks. Despite the lack of epidemiological data about fertility in female cancer survivors, there are known chemotherapy and radiation risks that delay pubertal development and cause gonadal dysfunction; this makes successful pregnancy in our patient even more exceptional. Female menstrual cycles become abnormal during treatment with alkylating agents but presumably normalize after completion of chemotherapy. ${ }^{[14]}$ Comprehensive data from the Childhood Cancer Survivor Study showed that infertility from alkylating agents such as ifosfamide occurred in a dose-dependent manner, and females were much less likely to become pregnant when receiving a cumulative ifosfamide dose between 55,759 and $192,391 \mathrm{mg} / \mathrm{m}^{2}$. Despite a cumulative ifosfamide dose over $86,000 \mathrm{mg} / \mathrm{m}^{2}$, our patient experienced no difficulties with conception. The Childhood Cancer Survivorship Study data also showed that compared to female siblings not exposed to alkylating agents, the relative risk (RR) of

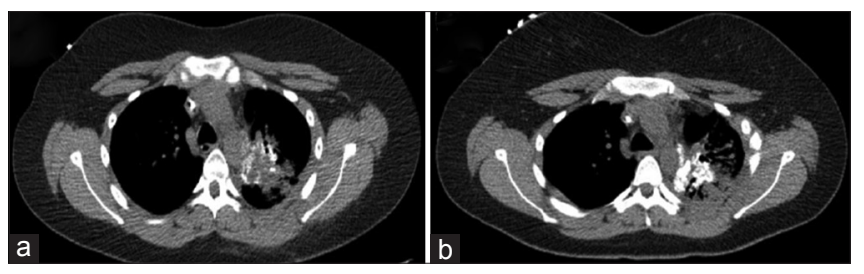

Figure 2: (a) Chest lesions per computed tomography before lexatumumab initiation. (b) After 20 cycles of lexatumumab, osteosarcoma lesions have become necrotic and calcified. No evidence of progressive disease 
becoming pregnant dropped to 0.72 . Infertility was more pronounced in patients $<24$ years of age (RR 2.92), even after adjusting for sociodemographic and behavioral risk factors. In addition, early menopause exacerbates difficulties with fertility. Exposure to chemotherapy not only increases the risk of miscarriages and early menopause but also potentially increases the risk of congenital deformities for the child. ${ }^{[15-18]}$ Our patient delivered a healthy boy without any deformity.

Limited treatment options for refractory or relapsed OS result in dismal prognosis. In this context, it is remarkable that our patient has survived for $>5$ years with stable disease. In the absence of complete resection of pulmonary metastasis, our patient received high-dose multiagent neoadjuvant and adjuvant chemotherapy, palliative radiation, and Phase 1 monoclonal antibody lexatumumab before disease stabilization. Tumor response and subsequent outcome argues for an aggressive approach without compromising the quality of life in patients with recurrent metastatic OS. Careful consideration of the biology of the tumor and implementation of novel biological agents are essential to further improve outcomes in patients with these tumors yielding poor prognosis. Furthermore, such agents may minimize the dose of alkylating agents that lead to gonadal dysfunction and infertility as the goal is not only for patients to survive but also to think ahead and find a means for survivors to also thrive.

\section{Financial support and sponsorship}

Nil.

\section{Conflicts of interest}

There are no conflicts of interest.

\section{References}

1. Allison DC, Carney SC, Ahlmann ER, Hendifar A, Chawla S, Fedenko A, et al. A meta-analysis of osteosarcoma outcomes in the modern medical era. Sarcoma 2012;2012:704872.

2. The European and American Osteosarcoma Study, Treatment. Available from: http://www.ctu.mrc.ac.uk/euramos/about_o_ treatment.asp. [Last accessed on 2013 Mar 13].

3. A Randomized Trial of the European and American Osteosarcoma Study Group to Optimize Treatment Strategies for Resectable Osteosarcoma Based on Histological Response to Pre-Operative Chemotherapy (IND\#12697). The Children's Oncology Group. 06 September, 2011. Available from: https:// www.members.childrensoncologygroup.org/Prot/AOST0331/ AOST0331DOC.pdf. [Last accessed on 2013 Jul 04].

4. Whelan J, Patterson D, Perisoglou M, Bielack S, Marina N, Smeland $\mathrm{S}$, et al. The role of interferons in the treatment of osteosarcoma. Pediatr Blood Cancer 2010;54:350-4.

5. Tabone MD, Kalifa C, Rodary C, Raquin M, Valteau-Couanet D, Lemerle J. Osteosarcoma recurrences in pediatric patients previously treated with intensive chemotherapy. J Clin Oncol 1994;12:2614-20.

6. Merchant MS, Geller J, Baird K, Chou AJ, Galli S, Charles A, et al. Phase I trial and pharmacokinetic study of lexatumumab in pediatric patients with solid tumors. J Clin Oncol 2012;30:4141-7.

7. Weigel B, Malempati S, Reid JM, Voss SD, Cho SY, Chen HX, et al. Phase 2 trial of cixutumumab in children, adolescents, and young adults with refractory solid tumors: A report from the Children's Oncology Group. Pediatr Blood Cancer 2014;61:452-6.

8. Choy E, Flamand Y, Balasubramanian S, Butrynski JE, Harmon DC, George S, et al. Phase 1 study of oral abexinostat, a histone deacetylase inhibitor, in combination with doxorubicin in patients with metastatic sarcoma. Cancer 2015;121:1223-30.

9. Grignani G, Palmerini E, Ferraresi V, D’Ambrosio L, Bertulli R, Asaftei SD, et al. Sorafenib and everolimus for patients with unresectable high-grade osteosarcoma progressing after standard treatment: A non-randomised phase 2 clinical trial. Lancet Oncol 2015;16:98-107.

10. Thompson PA, Drissi R, Muscal JA, Panditharatna E, Fouladi M, Ingle AM, et al. A phase I trial of imetelstat in children with refractory or recurrent solid tumors: A Children's Oncology Group Phase I Consortium Study (ADVL1112). Clin Cancer Res 2013;19:6578-84.

11. A Study of Bevacizumab, a Humanized Monoclonal Antibody against Vascular Endothelial Growth Factor (VEGF), in Combination With Chemotherapy for Treatment of Osteosarcoma. Available from: https://www.clinicaltrials.gov/ct2/ show/NCT00667342. [Last accessed on 2015 Jul 07].

12. Turner DC, Navid F, Daw NC, Mao S, Wu J, Santana VM, et al. Population pharmacokinetics of bevacizumab in children with osteosarcoma: Implications for dosing. Clin Cancer Res 2014;20:2783-92.

13. Grignani G, Palmerini E, Dileo P, Asaftei SD, D'Ambrosio L, Pignochino $\mathrm{Y}$, et al. A phase II trial of sorafenib in relapsed and unresectable high-grade osteosarcoma after failure of standard multimodal therapy: An Italian Sarcoma Group study. Ann Oncol 2012;23:508-16.

14. Osteosarcoma, American Cancer Society. Available from: http:// www.cancer.org/acs/groups/cid/documents/webcontent/003129pdf.pdf. [Last accessed on 2013 Mar 16].

15. Green DM, Kawashima T, Stovall M, Leisenring W, Sklar CA, Mertens AC, et al. Fertility of female survivors of childhood cancer: A report from the childhood cancer survivor study. J Clin Oncol 2009;27:2677-85.

16. Barton SE, Najita JS, Ginsburg ES, Leisenring WM, Stovall M, Weathers RE, et al. Infertility, infertility treatment, and achievement of pregnancy in female survivors of childhood cancer: A report from the Childhood Cancer Survivor Study cohort. Lancet Oncol 2013;14:873-81.

17. Longhi A, Pignotti E, Versari M, Asta S, Bacci G. Effect of oral contraceptive on ovarian function in young females undergoing neoadjuvant chemotherapy treatment for osteosarcoma. Oncol Rep 2003;10:151-5.

18. Hoshi M, Takami M, Ieguchi M, Aono M, Takada J, Oebisu N, et al. Fertility following treatment of high-grade malignant bone and soft tissue tumors in young adults. Mol Clin Oncol 2015;3:367-74. 